\title{
Mehr registrierte Fälle von Kindesmisshandlung
}

\section{Markus Wopmann}

Leiter der Fachgruppe Kinderschutz der schweizerischen Kinderkliniken

\section{Grosser Teil der Fälle erfasst}

Im Jahre 2013 wurden im fünften Jahr in Folge die Kinder erfasst, die wegen vermuteter oder sicherer Kindesmisshandlung ambulant oder stationär an einer schweizerischen Kinderklinik behandelt worden waren. Für die Erfassung standen die Daten von 18 der insgesamt 26 Kliniken (69\%) zur Verfügung. Die grossen und mittleren Kinderkliniken der Schweiz machten praktisch ausnahmslos an der Erfassung mit, keine Meldungen kamen von wenigen kleineren oder kleinsten Kinderabteilungen, so dass die von uns erfasste Gesamtzahl der Fälle einen grossen Teil der Fälle von Kindesmisshandlung darstellen sollte, die an den schweizerischen Kinderkliniken im Jahre 2013 behandelt worden waren.

\section{Einerseits gibt es eine bessere Erfassung der Fälle, andererseits eine erhöhte Sensibilisierung der behandelnden Fachpersonen.}

Korrespondenz: Dr. med. Markus Wopmann Chefarzt der Klinik für Kinder und Jugendliche Kantonsspital CH-5404 Baden

\section{Resultate}

Von den 18 Kliniken wurden insgesamt 1292 Fälle gemeldet, rund 13\% mehr als im Vorjahr. Eine so hohe Anzahl von Fällen wurde in den vergangenen fünf Jahren nie registriert, was einerseits sicher mit einer besseren Erfassung der Fälle, andererseits auch mit einer erhöhten Sensibilisierung der behandelnden Fachpersonen zu tun hat. Dass es sich um eine echte Zunahme der Fälle handelt, ist nicht ausgeschlossen, entspricht jedoch nicht den internationalen Vergleichsdaten. Die Aufteilung der Fälle in die verschiedenen Untergruppen ergab Folgendes:

\begin{tabular}{llr}
\hline Körperliche Misshandlung & 351 & $(27,2 \%)$ \\
\hline Vernachlässigung & 323 & $(25,0 \%)$ \\
\hline Psychische Misshandlung & 323 & $(25,0 \%)$ \\
\hline Sexueller Missbrauch & 281 & $(21,7 \%)$ \\
\hline Münchhausen-Stellvertreter-Syndrom & 14 & $(1,1 \%)$
\end{tabular}

Jedes vierte körperlich misshandelte Kind ist jünger als 2 Jahre, in der Gruppe der vernachlässigten Kinder ist sogar jedes zweite Kind jünger als 2 Jahre. Drei Kinder sind aufgrund der Misshandlung verstorben, alle waren jünger als 1 Jahr. Die Altersgruppe der 0- bis 1-jährigen Kinder war mit 245 Fällen (19\%) erneut am stärksten von irgendeiner Form von Kindesmisshandlung betroffen, $46 \%$ aller misshandelten Kinder sind jünger als 6 Jahre. Von der Gesamtzahl der betroffenen Kinder waren 45\% Knaben und 55\% Mädchen.
Geschlecht der Kinder

in den einzelnen Diagnosegruppen:

\begin{tabular}{|lll|} 
& \multicolumn{2}{c}{ Knaben Mädchen } \\
\hline Körperliche Misshandlung & $54 \%$ & $46 \%$ \\
\hline Vernachlässigung & $52 \%$ & $48 \%$ \\
\hline Psychische Misshandlung & $46 \%$ & $54 \%$ \\
\hline Sexueller Missbrauch & $24 \%$ & $76 \%$
\end{tabular}

Erneut recht ausgeglichene Geschlechterverteilung ausser beim sexuellen Missbrauch, bei dem die Mädchen dreimal häufiger betroffen sind als die Knaben.

\section{Sicherheit der Diagnose}

\begin{tabular}{lll} 
Sicher & 713 & $(55 \%)$ \\
\hline Wahrscheinlich & 270 & $(21 \%)$ \\
\hline Unklar & 308 & $(24 \%)$
\end{tabular}

Erneut war die Sicherheit der Diagnose bei der psychischen Misshandlung (70\%) und bei der körperlichen Misshandlung (60\%) am höchsten, bei vermuteter Vernachlässigung nur in der Hälfte der Fälle, bei vermutetem sexuellem Missbrauch in gut einem Drittel der Fälle.

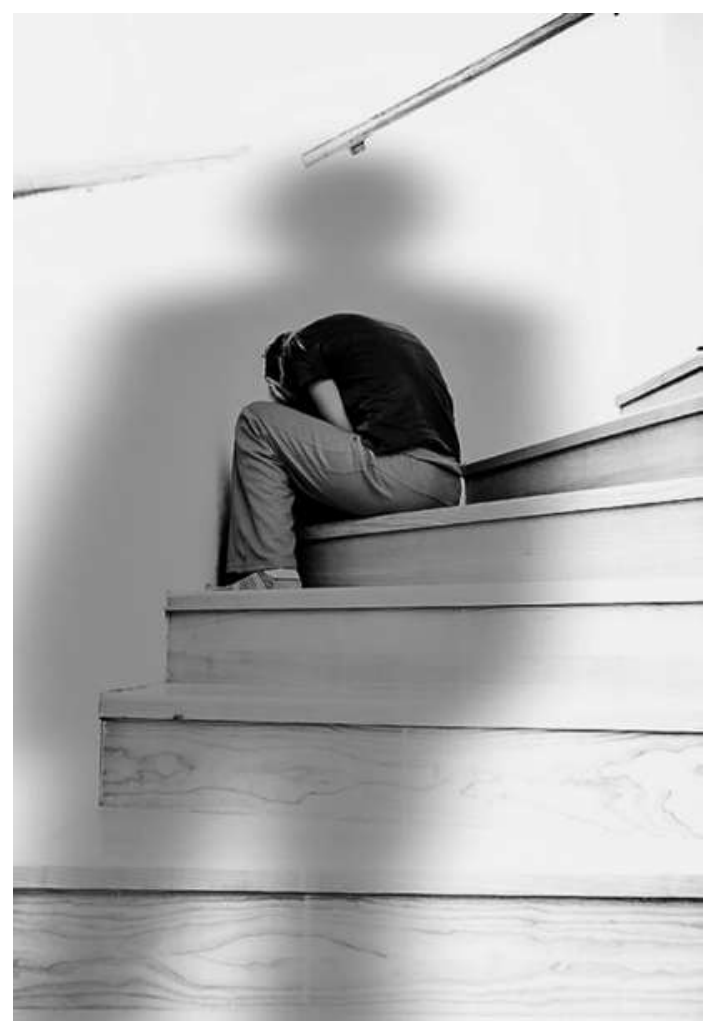

Körperliche Misshandlungen wurden in $75 \%$ der Fälle von engen Familienmitgliedern ausgeübt, sexueller Missbrauch in $42 \%$ der Fälle. 
Täterin/Täter: Beziehung zum Kind

$\begin{array}{lcr}\text { Familie } & 1008 & (78,0 \%) \\ \text { Bekannte/r des Kindes } & 177 & (13,7 \%) \\ \text { Fremdtäter } & 46 & (3,6 \%) \\ \text { Unbekannter Täter } & 61 & (4,7 \%)\end{array}$

Vernachlässigung und psychische Misshandlung wird praktisch immer im engsten Familienrahmen ausgeübt, körperliche Misshandlung in 75\% der Fälle, sexueller Missbrauch in $42 \%$ der Fälle.

\section{Täterin/Täter: Geschlecht}

\begin{tabular}{llr} 
Männlich & 614 & $(47,5 \%)$ \\
\hline $\begin{array}{l}\text { Weiblich } \\
\text { Männlich und weiblich } \\
\text { (meist Eltern gemeinsam) }\end{array}$ & 362 & $(28,0 \%)$ \\
\hline Unbekannt & 214 & $(16,6 \%)$ \\
\hline Keine Angabe & 97 & $(7,5 \%)$ \\
\hline
\end{tabular}

Im Falle eines sexuellen Missbrauches war der Täter in 83\% der Fälle männlich. Jeweils beide Elternteile wurden verantwortlich gemacht für 35\% der Fälle von Vernachlässigung, 18\% der Fälle von psychischer Misshandlung und $10 \%$ der Fälle von körperlicher Misshandlung.

\section{Täterin/Täter: Alter}

\begin{tabular}{|c|c|c|}
\hline Älter als 18 Jahre & 1101 & $(85,2 \%)$ \\
\hline Jünger als 18 Jahre & 110 & $(8,5 \%)$ \\
\hline $\begin{array}{l}\text { Jünger und älter als } 18 \text { Jahre } \\
\text { (mehrere Täter) }\end{array}$ & 6 & $(0,5 \%)$ \\
\hline Unbekannt & 97 & $(7,5 \%)$ \\
\hline Unbekanntes Alter / keine Angabe & 75 & $(6,0 \%)$ \\
\hline
\end{tabular}

Auch dieses Jahr sind die jugendlichen Täter vor allem beim sexuellen Missbrauch vertreten, wo sie 18,5\% der Fälle darstellen.

\section{Vormundschaftliche Massnahmen}

\begin{tabular}{lcc|}
$\begin{array}{l}\text { Durch eine andere Stelle bereits } \\
\text { eingeleitet }\end{array}$ & 253 & $(19,6 \%)$ \\
$\begin{array}{l}\text { Gefährdungsmeldung durch die } \\
\text { Kinderschutzgruppe gemacht }\end{array}$ & 331 & $(25,6 \%)$ \\
$\begin{array}{l}\text { Gefährdungsmeldung durch die } \\
\text { Kinderschutzgruppe empfohlen }\end{array}$ & 157 & $(12,2 \%)$
\end{tabular}

\section{Strafrechtliche Massnahmen}

\begin{tabular}{llr}
\hline Durch andere Stellen bereits eingeleitet & 168 & $(13,0 \%)$ \\
\hline Durch die Kinderschutzgruppe veranlasst 85 & $(6,6 \%)$ \\
$\begin{array}{l}\text { Durch die Kinderschutzgruppe } \\
\text { empfohlen }\end{array}$ & 44 & $(3,4 \%)$
\end{tabular}

\section{Fazit}

- Deutlich angestiegene Anzahl von registrierten Fällen von Kindesmisshandlung an schweizerischen Kinderkliniken, wobei, wie in den Jahren zuvor, vor allem die kleinen und kleinsten Kinder betroffen sind.

- Körperliche Misshandlung, psychische Misshandlung und Vernachlässigung sind praktisch gleich vertreten und kommen zudem häufig in Kombination untereinander vor. Von Seiten der Kinderschutzgruppen wird in jedem vierten Fall eine Meldung an die Kindes- und Erwachsenenschutzbehörde gemacht, in gut $6 \%$ der Fälle auch eine Strafanzeige. Dies widerspiegelt die Überzeugung, dass zum Schutz der Kinder oft behördliche Massnahmen nötig sind und Hilfe auf freiwilliger Basis nicht ausreicht.

- Die Tatsache, dass allein im letzten Jahr drei Kinder an den Folgen einer Kindesmisshandlung (nur registrierte Fälle an Kinderkliniken!) verstorben sind, unterstreicht den Schweregrad und die Wichtigkeit dieser Thematik. 